\title{
THE IMAGE OF UNITARY BORDISM IN UNORIENTED BORDISM-THE EQUIVARIANT \\ CASE
}

\author{
R. PAUL BEEM AND E. R. WHEELER
}

ABSTRACT. It is shown that the image of unitary $G$-bordism in unoriented $G$-bordism is the set of squares in unoriented $G$-bordism if $G$ is $Z_{2}$ and properly contains the squares if $G$ is an odd order finite group.

1. Statement of results. In [3], Milnor shows that the image of the reduction homomorphism from unitary to unoriented bordism is the same as the image of the squaring homomorphis $m$ from unoriented bordis $m$ into itself. We consider an equivariant version of this result. Specifically, if $U_{*}^{G}$ and $N_{*}^{G}$ denote, respectively, the unitary and unoriented equivariant bordism of smooth $G$ actions on closed manifolds (with unrestricted isotropy subgroups), $\rho$ : $U_{*}^{G} \rightarrow N_{*}^{G}$ denotes the reduction homomorphism which "forgets" the stably almost complex structure, and $\sigma: N_{*}^{G} \rightarrow N_{*}^{G}$ is defined by $\sigma(x)=x^{2}$, then

Theorem 1. If $G=Z_{2}$, then the image of $\rho$ equals the image of $\sigma$.

Theorem 2. If the order of $G$ is odd, then the image of $\sigma$ is properly contained in the image of $\rho$.

2. Proof of Theorem 1. Denote by $U_{*}(X)$ and $N_{*}(X)$, respectively, the unitary and unoriented bordism of the space $X$. Assigning to an involution on a manifold, the normal bundle to its fixed set gives rise to fixed point homomorphisms

$$
F: N_{*}^{Z}{ }^{2} \rightarrow \bigoplus N_{*}(B O(k)) \quad \text { and } \quad F^{u}: U_{*}^{Z}{ }^{2} \rightarrow \bigoplus U_{*}(B U(k)) \text {. }
$$

There are exact sequences:

$$
0 \rightarrow U_{*} \rightarrow U_{*}^{Z} \stackrel{F}{ }^{u} \bigoplus_{k=0}^{* / 2} U_{*-2 k}(B U(k)) \rightarrow \tilde{U}_{*-1}(B O(1)) \rightarrow 0
$$

Received by the editors October 18, 1973.

AMS (MOS) subject classifications (1970). Primary 57D85.

Key words and phrases. G-bordis m, bordism exact sequences. 


$$
0 \rightarrow N_{*}^{Z} \stackrel{F}{\longrightarrow} \bigoplus_{k=0}^{*} N_{*-k}(B O(k)) \rightarrow N_{*-1}(B O(1)) \rightarrow 0 .
$$

(See $[4$, Proposition 4] and $[5, \$ 1]$ for these sequences.)

Introduction of reduction homomorphisms gives a commutative diagram:

$$
\begin{aligned}
0 \rightarrow U_{*} & \rightarrow U_{*}^{Z_{2}} \stackrel{F^{u}}{\longrightarrow} \oplus U_{*-2 k}(B U(k)) \rightarrow \tilde{U}_{k-1}(B O(1)) \rightarrow 0 \\
& \left.\rho\right|_{Z_{2}} \stackrel{F}{\rightarrow} \bigoplus N_{*-k}(B O(k)) \rightarrow N_{*-1}(B O(1)) \rightarrow 0 .
\end{aligned}
$$

Assigning to a real $k$-plane bundle $E$ the total space of the projective space bundle of $E \oplus 1$ with the involution induced by $(+1) \oplus(-1)$ on $E \oplus 1$, induces a homomorphism $G: \bigoplus N_{*}(B O(k)) \rightarrow N_{*}^{Z}{ }^{2}$, which is a left inverse for $F$ and splits sequence (2) [2, (28.2)]. There is a similarly defined homomorphism $G^{u}: \bigoplus U_{*}(B U(k)) \rightarrow U_{*}^{Z}$, which of course is not a splitting homomorphism.

Clearly, there are products in $N_{*}^{Z}, \bigoplus N_{*}(B O(k)), U_{*}^{Z_{2}}$ and $\bigoplus U_{*}(B U(k))$ giving these modules the structure of algebras. Under these products, the homomorphisms $F$ and $F^{u}$ are algebra homomorphisms. From [1, (2.2)], we know that $\bigoplus U_{*}(B U(k))$ is the graded polynomial algebra over $U_{*}$ whose generators are the classes of the Hopf line bundles $\{[\gamma \rightarrow C P(n)]\}$. Also, $\bigoplus N_{*}(B O(k))$ is the graded polynomial algebra over $N_{*}$ on the classes of the nontrivial line bundles over the $R P(n),\{[\lambda \rightarrow R P(n)]\}$.

Just as in $[2,(24.2)]$, where it is shown that the normal bundle to the fixed set of an involution determines the bordism class of the underlying manifold, it can be shown that the normal bundle to the fixed set of a bundle involution together with the bundle restricted to that $f$ ixed set determine the underlying class in $N_{*}(B O(k))$ of the bundle with involution [4, Proposition 8]. In particular, the bundle $\gamma \rightarrow C P(n)$ with "conjugation" and the bundle $\lambda \times \lambda \rightarrow R P(n) \times R P(n)$ with the "twist" involution have the same fixed information, since in both cases the normal bundle to the fixed set is the tangent bundle over $R P(n)$ and the two-plane bundle restricted to the fixed set is the complexification of $\lambda$. Hence these bundles are bordant in $N_{*}(B O(2))$ and the image of $\hat{\rho}$ is equal to the image of squaring in $\bigoplus N_{*_{-k}}(B O(k))$.

For an $n$-tuple $w=\left(i_{1}, \cdots, i_{n}\right)$ of nonnegative integers, let $[R P(w), \lambda(w)]$ denote the bordism class of the $n$-plane bundle $\lambda \times \lambda \times \cdots \times \lambda \rightarrow R P\left(i_{1}\right) \times \cdots$ 
$\times R P\left(i_{n}\right)$, in $N_{m}(B O(n))$, where $m=i_{1}+\cdots+i_{n}$. We define $[C P(w), \gamma(w)]$ in $U_{2 m}(B U(n))$ similarly, using the bundles $\gamma \rightarrow C P\left(i_{j}\right)$. The collections $\{[C P(w), \gamma(w)]\}$ and $\{[R P(w), \lambda(w)]\}$ are, respectively, free $U_{*}$ and $N_{*} \bmod -$ ule bases for $\bigoplus U_{*_{-} 2 k}(B U(k))$ and $\bigoplus N_{*_{-} k}(B O(k))$. If $\Lambda$ is the line bundle over $R P(\lambda(w))$ defined by the sphere of $\lambda(w)$ and $\Gamma$ is the similarly defined (complex) line bundle over $C P(\gamma(w))$, then the class $[C P(\gamma(w)), \Gamma]$ is equal to the class of $[R P(\lambda(w)), \Lambda]^{2}$ in $N_{2 m-2}(B O(2))$. (A proof would aga in involve a comparison of the fixed set information of the twist involution on $\Lambda \times \Lambda$ and conjugation on $\Gamma$.)

To understand $F^{u}\left(U_{*}^{Z} Z_{2}\right)$ in $\bigoplus U_{*}(B U(k))$ one considers $\bar{U}_{*}^{Z}$, the bordism of involutions on unitary manifolds with codimension two fixed sets (possibly empty). There is an exact sequence:

$$
0 \rightarrow U_{*} \rightarrow \bar{U}_{*}^{Z_{2}} \stackrel{F^{u}}{\longrightarrow} U_{*-2}(B U(1)) \rightarrow \widetilde{U}_{*-1}(B O(1)) \rightarrow 0,
$$

which is a direct summand of (1) $[5, \S 1]$. The (disc, sphere) cofibration for the bundle $\gamma \otimes \gamma \rightarrow B U(1)$ yields a commutative diagram:

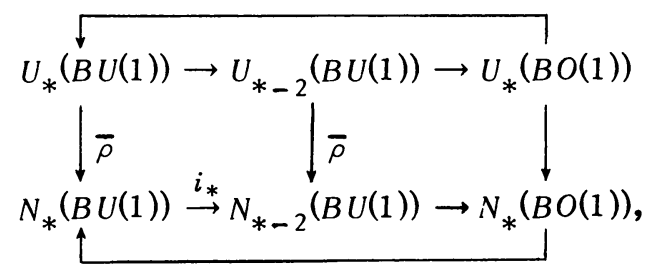

where the $\bar{\rho}$ 's again denote reduction homomorphisms. Since $i_{*}$ is zero on $Z_{2}$-cohomology, it is also zero on unoriented bordism. Further, (5) can be written

$$
0 \rightarrow U_{*} \rightarrow U_{*}(B U(1)) \rightarrow U_{*-2}(B U(1)) \rightarrow \widetilde{U}_{*-1}(B O(1)) \rightarrow 0
$$

and is isomorphic to sequence $(4)[5, \S 1]$. Hence $\rho F^{u}$ is zero as a homomorphism from $\bar{U}_{*}^{Z}$ to $N_{*}(B O(2))$.

Now, if $d=\Sigma\left[M_{k}, E_{k}\right]$ is in $\bigoplus U_{*-2 k}(B U(k))$ and $\Gamma_{k}$ denotes the canonical complex line bundle over $C P\left(E_{k}\right)$ (i.e., $\Gamma_{k}$ is a subbundle of $E_{k} \times$ $\left.C P\left(E_{k}\right)\right)$, then $F^{u} G^{u}(d)=d+\Sigma\left[C P\left(E_{k}\right), \Gamma_{k}\right]$. Defining the homomorphism $C P(\quad): \bigoplus U_{*-2 k}(B U(k)) \rightarrow U_{*-2}(B U(1))$ by $C P\left(\Sigma\left[M_{1}, E_{k}\right]\right)=\Sigma\left[C P\left(E_{k}\right), \Gamma_{k}\right]$, we have that $F^{u} G^{u}(d)=d+C P(d)$. Therefore, for each $d$ in $\bigoplus U_{*_{-}-2 k}(B U(k))$, there is some $c$ in $U_{*_{-}-2}(B U(1))$ with $d+c$ in the image of $F^{u}$. Moreover, according to the above, $c$ is unique modulo the kernel of $\hat{\rho}$. Similarly, if 
$R P(\quad): \bigoplus N_{*_{-k}}(B O(k)) \rightarrow N_{*_{-}}(B O(1))$ is defined by $R P\left(\Sigma\left[M_{k}, E_{k}\right]\right)=$ $\Sigma\left[R P\left(E_{k}\right), \Lambda_{k}\right]$, where $\Lambda_{k}$ is the canonical bundle over $R P\left(E_{k}\right)$, then $F G(b)$ $=b+R P(b)$. Hence, for each $b$ in $\bigoplus N_{*_{-} k}(B O(k))$ there is a unique (since $G$ is a splitting homomorphism) $a$ in $N_{*-1}(B O(1))$ with $a+b$ in the image of $F$.

Now let $x$ be in $N_{n}^{Z_{2}}$. $F(x)$ can be written uniquely as $a+b$ with $a$ in $N_{n-1}(B O(1))$ and $b$ in $\bigoplus_{k \neq 1} N_{n-k}(B O(k)) . F\left(x^{2}\right)=a^{2}+b^{2}$. There is a $d$ in $\bigoplus_{k \neq 1} U_{2 n-2 k}(B U(k))$ with $\hat{\rho}(d)=b^{2}$. Let $c=C P(d)$. Clearly $\hat{\rho}(C P(d))$ $=R P(b)^{2}$. Since $d+c=F^{u}(y)$ for some $y$ in $U_{2 n}^{Z_{2}}, \rho(y)=x^{2}$. Hence the image of $\rho$ contains the image of $\sigma$.

Conversely, if $y$ is in $U_{2 n}^{Z_{2}}$ and $F^{u}(y)=c+d$, where $c$ is in $U_{2 n-2}(B U(1))$ and $d$ is in $\bigoplus_{k \neq 1} U_{2 n-2 k}(B U(k))$, then $\hat{\rho}(d)=b^{2}$, where $b$ is in $\bigoplus_{k \neq 1} N_{n-k}(B O(k))$. Let $a=R P(b)$. Then

$$
\hat{\rho} F^{u}(y)=\hat{\rho}(d+C P(d))+\hat{\rho}(c-C P(d))=a^{2}+b^{2} .
$$

If $F(x)=a+b, x^{2}=\rho(y)$ and the reduction of each unitary involution is a square.

3. Proof of Theorem 2. In [7, Proposition 3] it is shown that the reduction homomorphism from $U_{*}^{G} \otimes_{U_{*}} N_{*}$ to $N_{*}^{G}$ is an epimorphism for groups $G$ of odd order. Hence for $a$ in $N_{*}^{G}$ there is an $x=\Sigma\left[M_{i}, T_{i}\right] \otimes\left[P_{i}\right]$ in $U_{*}^{G} \otimes_{U_{*}} N_{*}$ which reduces to $a$. However $x^{2}=\Sigma\left[M_{i}, T_{i}\right]^{2} \otimes\left[P_{i}\right]^{2}$ and, from Milnor's result, $\left[P_{i}\right]^{2}$ is the reduction of a unitary class. Hence $x^{2}$ is in $U_{*}^{Z} Z_{2}$ and squares are contained in the image of $\rho$.

To see that the containment is proper, one notes that Stong $[6, \S 12]$ indicates that for a group $G$ of odd order the bordism class of a $G$-manifold is determined by the collection of the fixed sets with their normal bundles of all subgroups of $G$. Since $G$ is odd, these bundles are all even dimensional. It follows that the normal bundle to a fixed set of any subgroup of $G$ acting on the square of a $G$-manifold has dimension at least four.

If $G$ is $Z_{p}$, the finite field of order $p, p$ odd, there is an exact sequence:

$$
0 \rightarrow U_{*} \rightarrow U_{*}^{Z}{ }^{Z} \stackrel{F^{\prime}}{\longrightarrow} \bigoplus U_{*}\left(B U\left(t_{1}\right) \times \cdots \times B U\left(t_{p-1}\right)\right) \rightarrow \widetilde{U}_{*}\left(B Z_{p}\right) \rightarrow 0,
$$

which arises from the relation between free and unrestricted bordism. Let $x=[C P(2), \gamma]$, which does not bord unorientably. Since $\widetilde{U}_{*}\left(B Z_{p}\right)$ is $p$-torsion, there is a $\left[M, Z_{p}\right]$ in $U_{*}^{Z} p$ and an odd integer $k$ with $F^{\prime}\left(\left[M, Z_{p}\right]\right)=k x$. 
The reduction of $\left[M, Z_{p}\right]$ cannot be a square since the fixed set of $Z_{p}$ must have codimension two.

For an arbitrary group $G$ of odd order, there is some odd prime $p$ with $Z_{p}$ as subgroup. If $\left[M, Z_{p}\right]$ is the pair described above, the extension of $\left(M, Z_{p}\right)$ to a manifold with $G$ action (i.e., the pair $\left.\left(M \times{ }_{Z_{p}} G, 1 \times G\right)\right)$ gives a class in $U_{*}^{G}$ which cannot be a square.

\section{BIBLIOGRAPHY}

1. P. :E. Conner, The bordism class of a bundle space, Michigan Math. J. 14 (1967), 289-303. MR 37 \#3579.

2. P. E. Conner and E. E. Floyd, Differentiable periodic maps, Ergebnisse der Math. und ihrer Grenzgebiete, N. F. Band 33, Academic Press, New York; SpringerVerlag, Berlin, 1964. MR 31 \#50.

3. J. W. Milnor, On the Stiefel-Whitney numbers of complex manifolds and of spin manifolds, Topology 3 (1965), 223-230. MR 31 \#207.

4. R. E. Stong, Bordism and involutions, Ann. of Math. (2) 90 (1969), 47-74. MR 39 \#3503.

5. - Complex and oriented equivariant bordism, Topology of Manifolds (Proc. Inst., Univ. of Georgia, Athens, Ga., 1969), Markham, Chicago, Ill., 1970, pp. 291-316. MR $42 \# 8521$.

6. - Unoriented bordism and actions of finite groups, Mem. Amer. Math. Soc. No. 103 (1970) MR 42 \#8522.

7. E. R. Wheeler, Localizing equivariant bordism, Proc. Amer. Math. Soc. (to appear).

DEPARTMENT OF MATHEMATICS, UNIVERSITY OF PENNSYLVANIA, PHILADELPHIA, PENNSYLVANIA 19104

DEPARTMENT OF MATHEMATICS, NORTHERN KENTUCKY STATE COLLEGE, HIGHLAND HEIGHTS, KENTUCKY 41076 